\title{
VERTICAL SCALES AND SHEAR STRESSES IN WAVE BOUNDARY LAYERS OVER MOVABLE BEDS.
}

\author{
Peter Nielsen \& Paul A Guard
}

\begin{abstract}
Unified scaling rules are provided for smooth and rough wave boundary layers. It is shown that the rough equivalent of the smooth, or viscous, vertical scale $\sqrt{2 v / \omega}$, the Stokes' length, is $\sqrt{0.008 r A}$, where $r$ is the Nikuradse roughness and $A$ is the near-bed semi excursion of the wave motion. Realizing this equivalence of viscous and rough scales a unified description in the style of Colebrook's (1939) formulae for steady flow friction can be devised based on the unified vertical scale $\mathrm{z}_{1}=\sqrt{2 v / \omega}+\sqrt{0.008 \mathrm{rA}}$. That is, unified smooth and rough wave friction factor formulae in the form $f_{\mathrm{w}}=f_{\mathrm{w}}\left(\mathrm{z}_{1} / \mathrm{A}\right)=f_{\mathrm{w}}([\sqrt{2 v / \omega}+\sqrt{0.008 r A}] / \mathrm{A})$ can be used with adequate accuracy. A general procedure is given for deriving $\mathrm{z}_{1}$ from velocity data including data from mobile bed experiments, which enable determination of the equivalent Nikuradse roughness from these experiments. Presently available sheet flow data show a velocity structure, which corresponds to a Nikuradse roughness $r$ of the order 50 to 100 grain diameters. Instantaneous shear stresses $\tau(\mathrm{z}, t)$ derived through the usual momentum integral from sheet flow experiments show that $\tau$ varies strongly through the sheet flow layer with the value at the lowest level of sediment motion being 2 to 3 times the value at the undisturbed bed level. The corresponding Nikuradse roughnesses are about $2.5 d_{50}$ corresponding to the undisturbed bed level and $100 d_{50}$ for the stress at the lowest level of sediment motion. With this strong variation of $\tau$ through the layer of moving sediment, it is not at all obvious what should be understood by THE BED SHEAR STRESS in the context of wave sediment transport.
\end{abstract}

Key words: wave boundary layers, hydraulic roughness, sheet flow, boundary layer structure

\section{INTRODUCTION}

Detailed data has accumulated over the last twenty odd years to the extent that we now have a reasonably clear picture of the velocity structure in oscillatory boundary layers over mobile sediment beds. Correspondingly, we are now able to refine boundary layer models of various forms. In the present paper we shall consider the analytical models which have a degree of similarity with the smooth laminar solution as opposed to time dependent log-law fits. That is, we consider models which are based on a complex velocity defect function $D=D(z)$ for each simple harmonic velocity component.

\section{DEFECT FUNCTION BOUNDARY LAYER MODELS}

We shall base our discussion of oscillatory boundary ayers on velocity defect models which have a degree of similarity with the smooth laminar solution for simple harmonic flow, i e, flows corresponding to a free stream velocity in the form $u_{\infty}(t)=A \omega \mathrm{e}^{\mathrm{i} \omega t}$ :

$$
\begin{aligned}
u(z, t) & =A \omega[1-D(z)] \mathrm{e}^{\mathrm{i} \omega t} \\
& =A \omega\left[1-\exp \left(-[1+\mathrm{i}] \frac{z}{\sqrt{2 v / \omega}}\right)\right] \mathrm{e}^{\mathrm{i} \omega t}
\end{aligned}
$$

Where $u(z, t)$ is local velocity, $A$ is the free stream semi excursion above the boundary layer, $\omega$ the angular frequency, $D=D(\mathrm{z})$ the complex velocity defect function and $v$ the kinematic viscosity.

\section{Determination of $z_{1}$ from velocity measurements}

Simple harmonic oscillatory boundary layers, and the individual harmonic components of more complicated periodic flows over movable as well as fixed beds can be analogously described by

School of Civil Engineering, The University of Queensland, Brisbane, AUSTRALIA 4072, Contact: p.nielsen@uq.edu.au 


$$
\begin{aligned}
u(z, t) & =A \omega[1-D(z)] \mathrm{e}^{\mathrm{i} \omega t} \\
& =A \omega\left[1-\exp \left(-[1+\alpha \mathrm{i}]\left(\frac{z}{z_{1}}\right)^{p}\right)\right] \mathrm{e}^{\mathrm{i} \omega t}
\end{aligned}
$$

See examples in Nielsen (1985) and Nielsen (1992) pp 44-51.

The expression (2) means that the vertical scale $\mathrm{z}_{1}$ is equal to the level at which $\ln |D|=-1$, and this enables determination of $\mathrm{z}_{1}$ from measured velocity data as shown in the figures of Nielsen (1992) pp 44-45.

\section{The rough flow equivalent of the Stokes' length}

For fully rough turbulent conditions the laminar vertical scale $\sqrt{2 v / \omega}$ is found to be replaced by $0.09 \sqrt{r A} \approx \sqrt{0.008 r A}$. This is borne out by the data in Figure 1

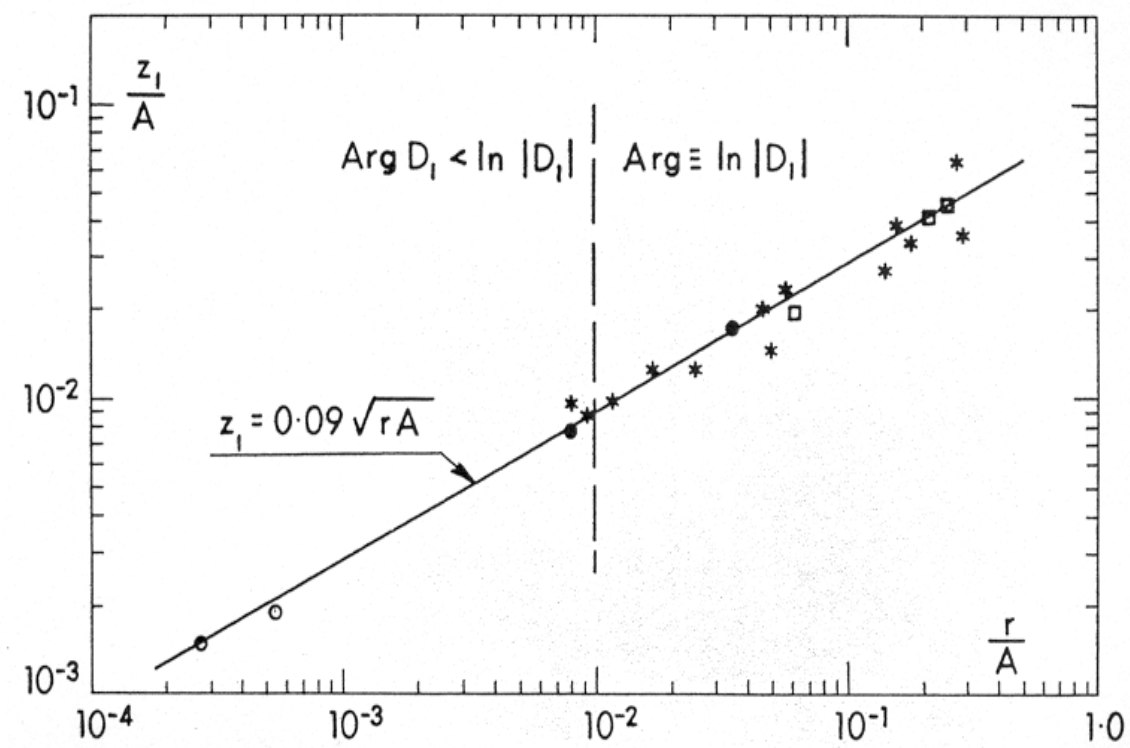

Figure 1: Experimental relationship between $z_{1}$ and $r, A$ from fixed bed experiments with roughness known from steady log-law fits. Legend: o Jensen et al (1989) 3D roughness, • Jonsson \& Carlsen (1976) strip roughness, $\square$ van Doorn (1982) strip roughness, * Sleath (1987) 3D roughness. After Nielsen (1992).

Hence, in the style of Colebrook (1939) for steady flows, the combined effects of viscosity and roughness on the structure of oscillatory flows can be expressed by the vertical scale:

$$
\mathrm{z}_{1}=\sqrt{2 v / \omega+0.008 r A}
$$

Eddy viscosity for turbulent oscillatory flows

In so far as $2 v / \omega \sim 0.008 r A$ we get the simple eddy viscosity estimate

$$
v_{t}=0.004 r A \omega \quad \text { for } r / A \gtrsim 0.01
$$


which applies when $\operatorname{Arg}\{D\} \approx \ln |D|$ corresponding to $\alpha=1$ in Equation (2). Based on available experimental data, Nielsen (1992) suggested that the relative roughness criterion for the validity of (4) is $r / A \gtrsim 0.01$. For smaller $\mathrm{z}_{1} / A$ a phase shift is observed between velocity gradients and momentum transfer corresponding to the eddy viscosity being complex, cf Nielsen (1992) pp 32-33.

\section{FRICTION FACTORS BASED ON VISCOSITY AND ROUGHNESS COMBINED}

Noting that $\sqrt{0.008 r A}$ is the "rough flow equivalent" of the smooth, viscous $\sqrt{2 v / \omega}$ suggests that friction factor formulae in the form

$$
f_{w}=f_{w}\left(\frac{z_{1}}{A}\right)=f_{w}\left(\frac{\sqrt{2 v / \omega}+\sqrt{0.008 r A}}{A}\right)
$$

should work with reasonable accuracy.

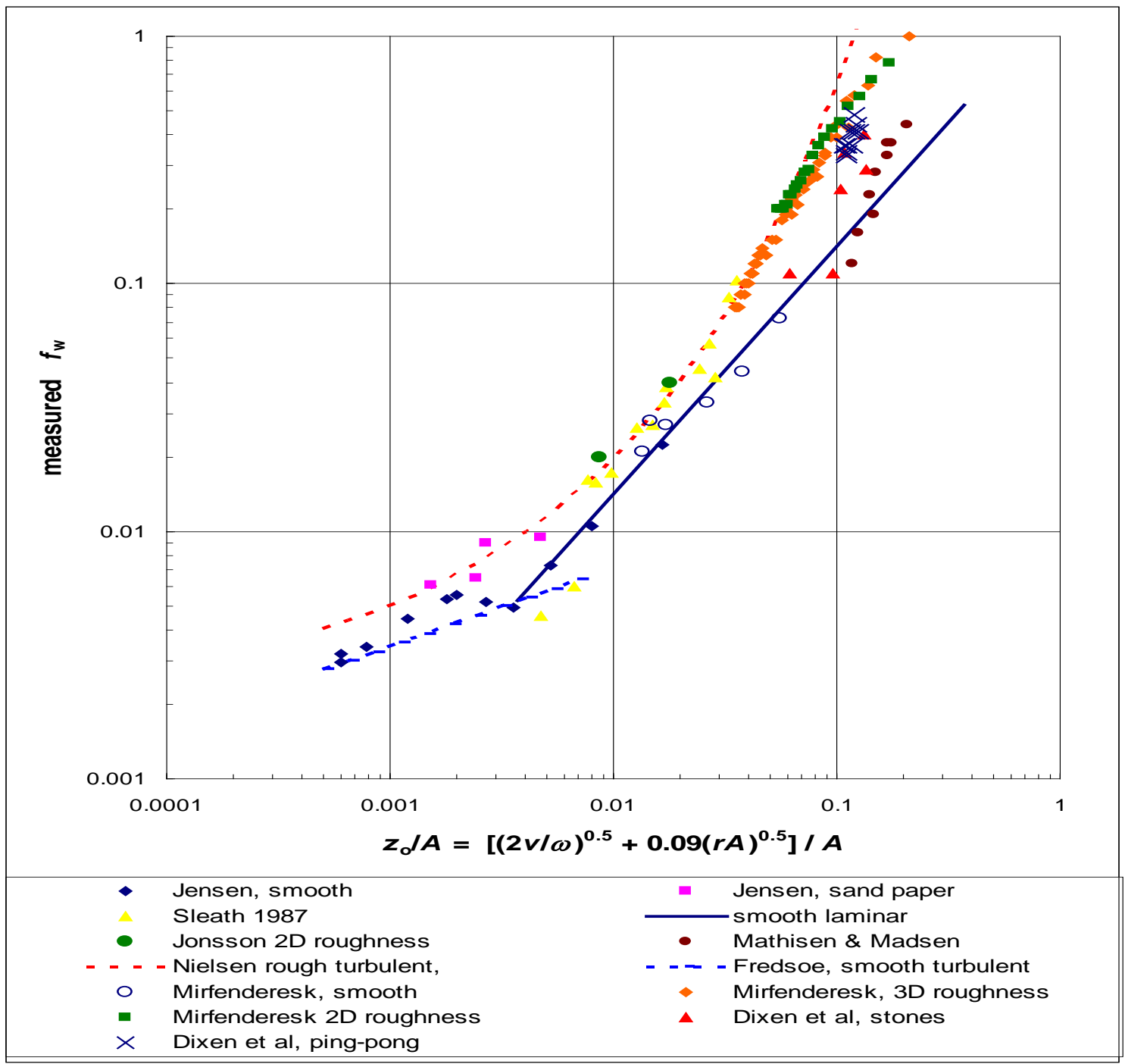

Figure 2: Measured wave friction factors plotted against $\mathrm{z}_{1} / A$ where $\mathrm{z}_{1}$ represents the combined effects of viscosity and Nikuradse roughness. The data includes smooth as well as rough flow conditions. The straight lines are friction factor formulae for laminar and fully turbulent smooth flows and the curve is the empirical fit by Nielsen (1992) p 25 to rough flow data. 


\section{Friction factor magnitude}

Figure 2 shows the extend to which the magnitude of the peak bed shear stress can be predicted on the basis of $\frac{Z_{1}}{A}=\frac{\sqrt{2 v / \omega}+\sqrt{0.008 r A}}{A}$ for smooth and rough flows alike.

That is, whether the traditional wave friction factor defined by $\tau_{\max }=\frac{1}{2} \rho f_{w}(A \omega)^{2}$ is of the form (5).

Improved overall fits may be obtained by combining the viscous and rough scales in more elaborate ways than the simple adding, e g by using

$$
z_{1}=\left(\sqrt{2 v / \omega}^{p}+\sqrt{0.008 r A}^{p}\right)^{\frac{1}{p}}
$$

with the power $p$ tanking some optimal value.

The phase lead of the bed shear stress ahead of the free stream velocity

Laminar flow theory shows that the bed shear stress in a smooth laminar flow leads the free stream velocity by 45 degrees, and experiments show that the phase lead is somewhat smaller for smooth turbulent flows, see e g, Fredsøe \& Deigaard (1992) p 32. For smooth flows there is no ambiguity in the sense that the level, at which THE bed shear stress acts is obviously the level of the flat bed, left panel in Figure 3.

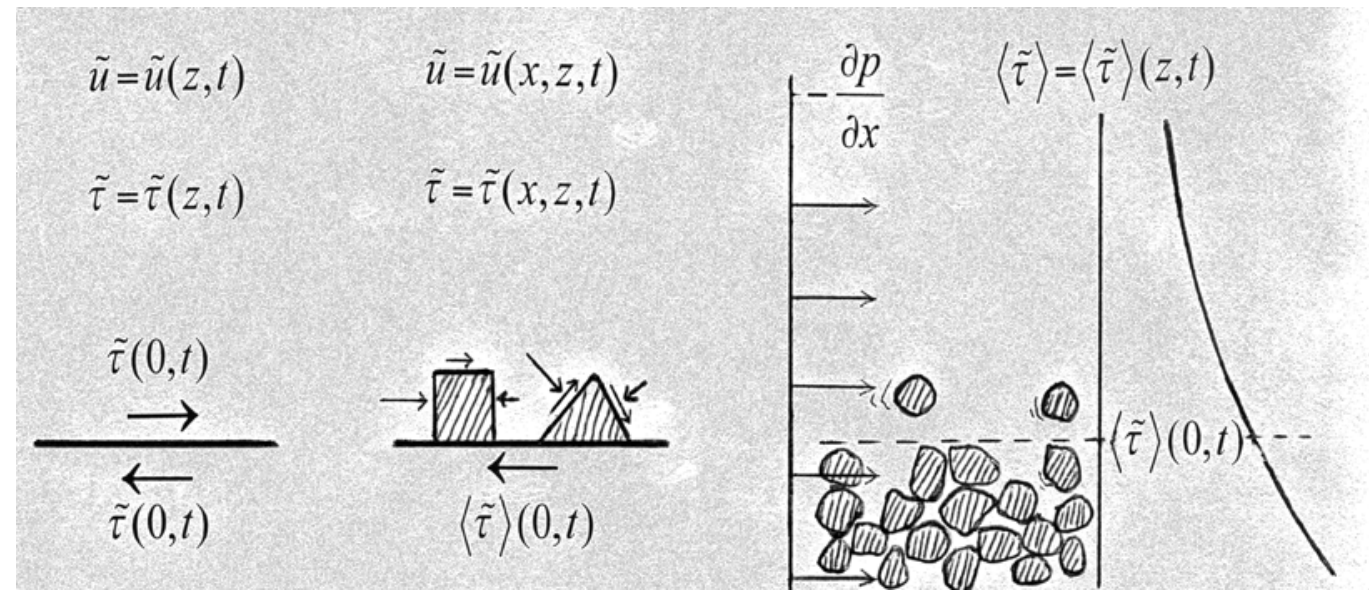

Figure 3: Shear stress distributions $\tilde{\tau}(x, z, t)$ and "bed shear stresses" on three different types of beds: left, a flat impermeable bed; middle, a rough impermeable bed and right a movable sand bed. For the left-hand scenario, smooth bed, the definition of THE bed shear stress is obvious.

The middle scenario with a rough impermeable bed is more complicated. There are normal stresses and tangential shear stresses with all sorts of directions around the roughness elements. The concept of a total bed shear stress defined as the total horizontal force per unit area only exists in a horizontally averaged sense but may be determined with a shear plate, which covers a large number of roughness elements. Many such measurements were carried out by Riedel (1972) as reported by Kamphuis (1975) and by Mirfenderesk \& Young (2003). For the movable bed scenario, it is not obvious at what level to put the Shear plate and correspondingly, the definition of THE bed shear stress is not obvious.

Instead of measuring $\langle\tilde{\tau}(0, t)\rangle$ with a shear plate, one might perhaps fit instantaneous log profiles to $\tilde{u}(z, t)=\langle\tilde{u}(x, z, t)\rangle$ and thus obtain $\tau_{\log }(t)$. But, what shear plate level would this correspond to? 
Just as the shear stress magnitude depends on the shear plate level so does the phase lead of the shear stress. Putting the shear plate one grain-layer lower will lead to a greater phase lead because the horizontal pressure gradient, which acts on that extra grain-layer, leads the free stream velocity by $90^{\circ}$.

Figure 4 shows phase leads measured in a variety of ways

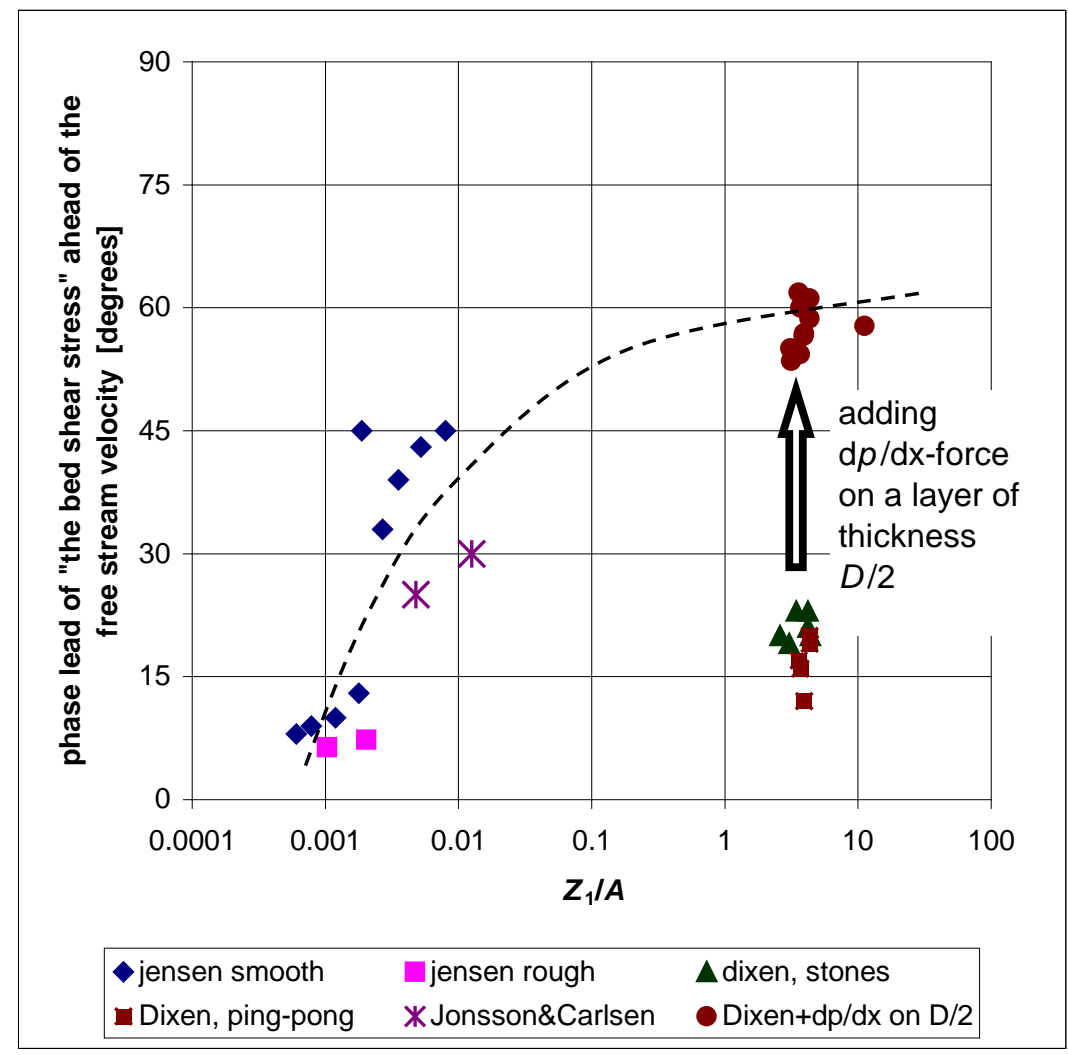

Figure 4: Phase lead of "bed shear stresses" ahead of the free stream velocity measured by hot film «; $\log$ fits $\square, \square, \boldsymbol{\Lambda}$; momentum integral * .

The very rough $(r / A \sim 1)$ measurements of Dixen et al do not follow the trend of the rest of the data unless and extra $\mathrm{d} p / \mathrm{d} x$ contribution, corresponding to half a grain diameter is added.

\section{HYDRAULIC ROUGHNESS OF FLAT MOVABLE BEDS IN SHEET FLOW}

Fitting of

$$
\ln |D|=-\left(\frac{z}{z_{1}}\right)^{p}
$$

to experimental data as shown by Nielsen (1992) pp 44-45 and subsequently getting the roughness by solving Equation (3) for $r$ can yield the equivalent Nikuradse roughness for any bed including movable beds at sheet flow conditions. Figure 5 shows $-\ln |D|$ vs z for sheet flow velocity data obtained by O’Donoghue et al (2004) with a $2^{\text {nd }}$ order Stokes' type of flow in an oscillating water tunnel.

It is interesting that, while a laminar flow (or a linear eddy viscosity model) would give the 2nd harmonic a smaller vertical scale (smaller by a factor $\sqrt{2}$ according to $z_{1}=\sqrt{2 v / \omega}$ ) compared with the 
fundamental mode, the 1 st and $2^{\text {nd }}$ harmonics of the turbulent flow in Figure 5 shows very similar vertical scales.

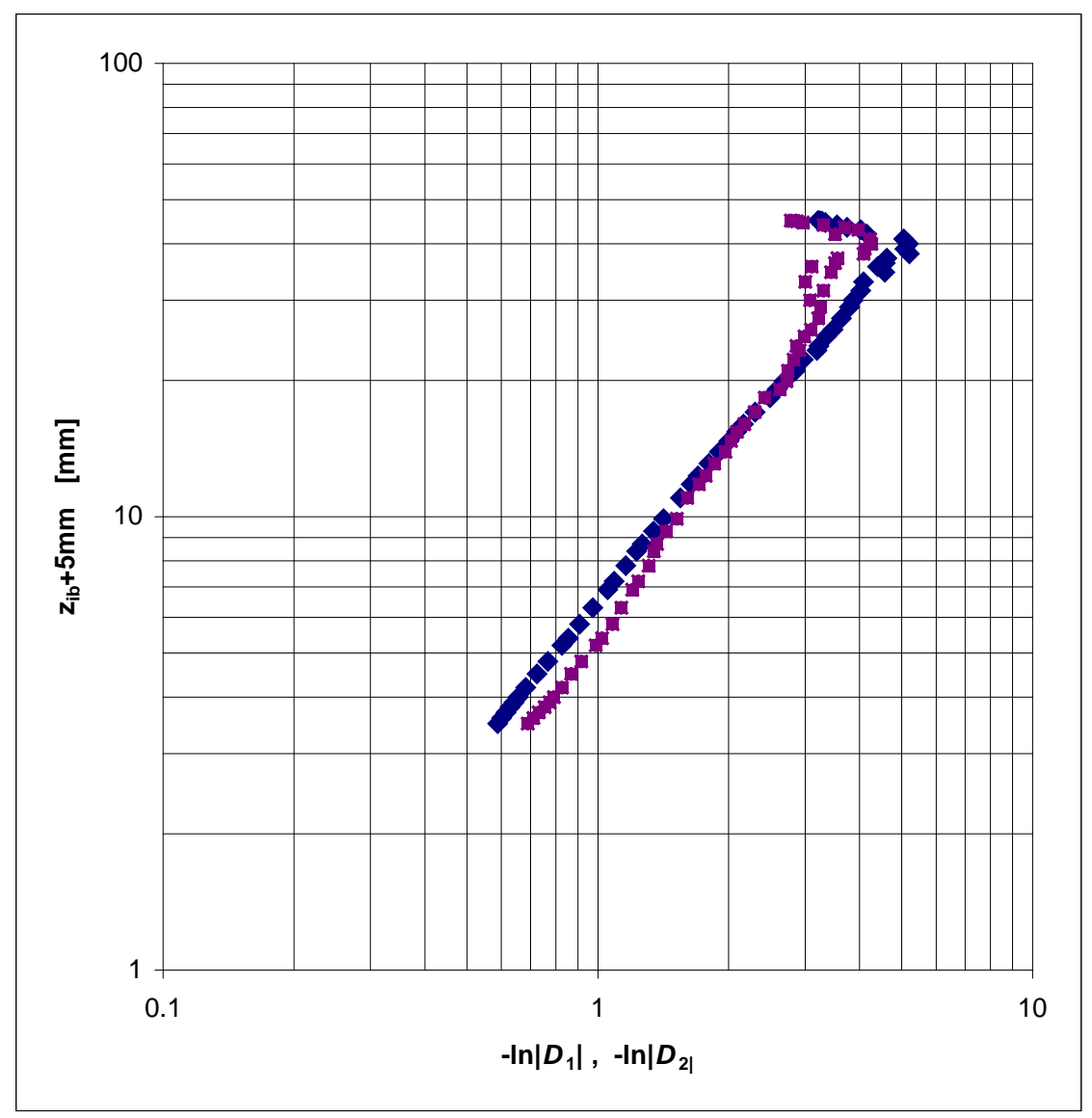

Figure 5: Velocity defect functions for the $1^{\text {st }}$ and $2^{\text {nd }}$ harmonics plotted in terms of $-\ln |D|$ for sheet flow data by O'Donoghue et al (2004), $T=6 \mathrm{~s}, A_{1}=1.13 \mathrm{~m}, d_{50}=0.15 \mathrm{~mm}$.

Placing the origin of the vertical axis $5 \mathrm{~mm}$ below the initial bed level, $\mathrm{z}_{\mathrm{ib}}$, has given the best straight line for the primary harmonic. Similar origin choices are used in log-law fitting. Hence, one can perhaps say that, the theoretical bed level is, in this sheet flow case, $5 \mathrm{~mm}$ below the initial bed level. Based on the first harmonic $\downarrow$, one finds $z_{1}=6.4 \mathrm{~mm}$ and hence via Equation (3) $r \approx 4.3 \mathrm{~mm} \approx 29 d_{50}$. This value is of course somewhat dependent of the choice of "theoretical bed level" just like when $r$ is found from loglaw fits to steady flow data. For the simple harmonic experiment from which $D(\mathrm{z})$ and friction factor data are shown in Figure 6 below a straight line fit, in the style of Figure 5, with the theoretical bed $9 \mathrm{~mm}$ below $\mathrm{z}_{\mathrm{ib}}$ gives $\mathrm{z}_{1} \approx 11.2 \mathrm{~m}$ leading to $r \approx 12.8 \mathrm{~mm} \approx 86 d_{50}$. As a rule of thumb, one can perhaps then say that the velocity structure as given by Equation (1), in typical oscillatory sheet flows correspond to a hydraulic roughness of the order $50 d_{50}$.

\section{SHEAR STRESSES IN SHEET FLOW LAYERS}

Local shear stresses $\tau(z, t)$ derived from a set of oscillatory flow data over a mobile bed through the momentum integral

$$
\tau(z, t)=\rho \int_{z}^{\infty} \frac{\partial}{\partial t}\left(u_{\infty}-u\right) d z^{\prime}
$$

Gives the picture in Figure 6. 

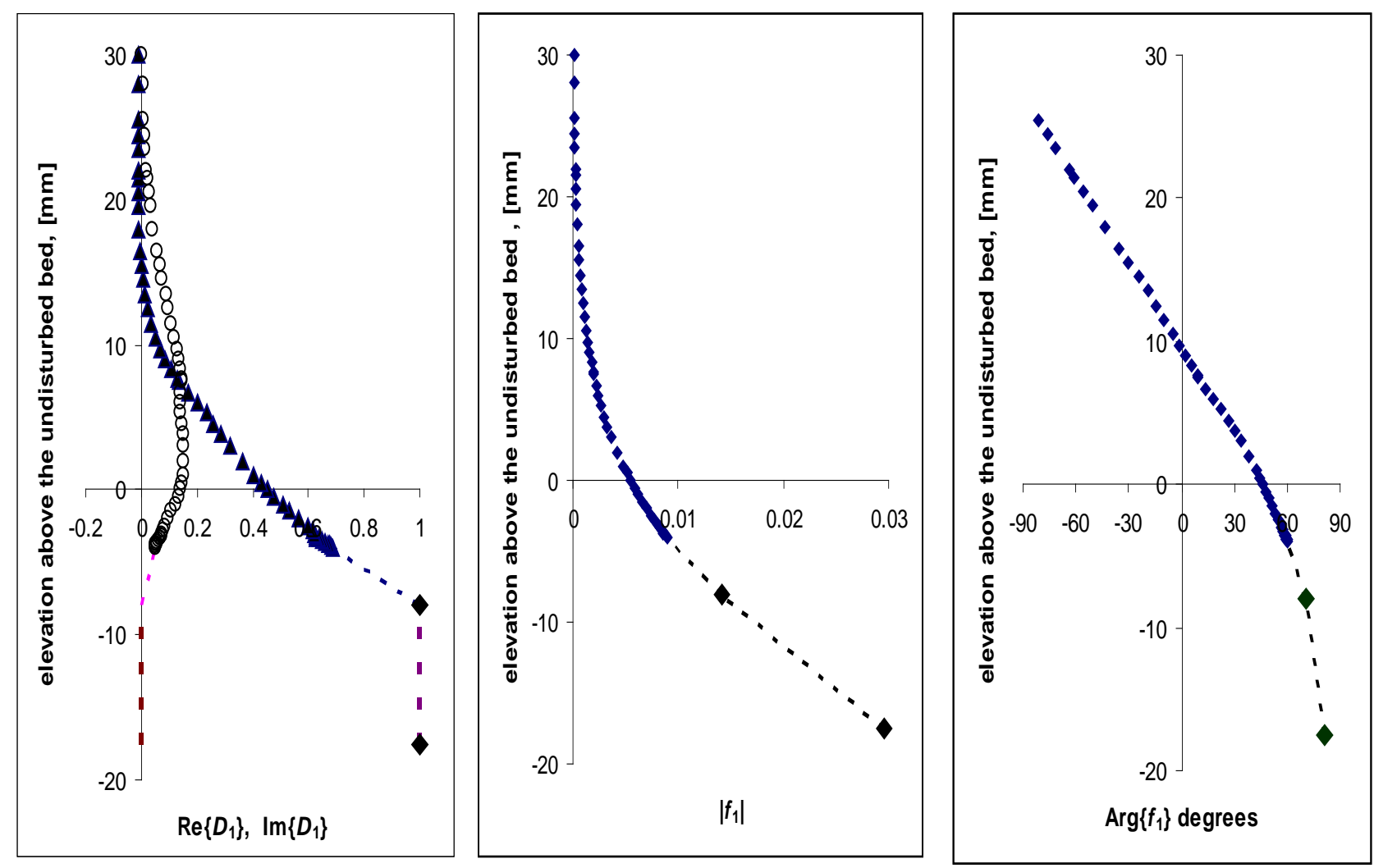

Figure 6: Left: Velocity defect function $D_{1}(z)$ (in terms of its real and imaginary parts). Center: magnitude of the friction factor $f_{1}(\mathrm{z})$ corresponding to the local shear stress magnitude and right: argument of $f_{1}(\mathrm{z})$ corresponding to the phase lead of the local shear stress relative to the free stream velocity. Data from O'Donoghue et al (2004), $T=6 \mathrm{~s}, A=1.2 \mathrm{~m}, d_{50}=0.15 \mathrm{~mm}$. $\mathrm{Z}=0$ is the undisturbed bed level.

The data indicates (partly by extrapolation to the level where $D=1$ ) that the sediment moves down to about $8 \mathrm{~mm}$ below the undisturbed bed level and that the shear stress varies by more than a factor two through the moving bed layer, $-8 \mathrm{~mm}<\mathrm{z}<0$. This restates the question as to what might be meant by "THE bed shear stress" and, which if any, single shear stress value can be used to determine the sediment motion.

\section{CONCLUSIONS}

Due to the strong horizontal pressure gradients which are present in vigorous wave generated flows, shear stresses vary rapidly with elevation, to the extent that the shear stress at the bottom of a sheet flow layer will be 2 to 3 times that at the undisturbed bed level. Correspondingly, the hydraulic roughness which corresponds to these stresses are widely different. Guard (2010) found that the stress at the initial bed level corresponds to a roughness of the order $2.5 d_{50}$, while that at the bottom of the moving layer corresponds to a roughness of the order $100 d_{50}$. It is therefore not obvious, which stress is the most appropriate to use in sediment transport calculations. This has in fact been addressed by recent sheet flow sediment transport models like that of Nielsen (2006), which incorporates the free stream acceleration which is proportional to $-\mathrm{d} p / \mathrm{d} x$ as well as a streaming related stress in order to model sediment transport under "real waves".

Investigations into oscillatory boundary layers over fixed beds with known roughness by Nielsen $(1985,1992)$ showed that the rough flow equivalent of the viscous vertical scale: the Stokes' length $\sqrt{2 v / \omega}$ is $\sqrt{0.008 r A}$, i e, the bed roughness $r$ and the free stream semi excursion $A$ contribute equally to the vertical scale of rough, oscillatory boundary layer flows. 
A Colebrook style combined vertical scale incorporating both viscous and roughness effects is therefore $\mathrm{z}_{1}$ $=\sqrt{2 v / \omega}+\sqrt{0.008 r A}$. The equivalence $2 v / \omega \sim 0.008 r A$ of the viscous and rough turbulent scales imply the simple rough turbulent eddy viscosity formula $v_{t}=0.004 r A \omega$ for $r / A \gtrsim 0.01$.

\section{REFERENCES}

Colebrook, C F (1939): Turbulent flow in pipes with particular reference to the transition region between rough and smooth pipe laws. J Inst Civ Eng, Nos 1-4, pp 135-156 + tables.

Dixen, M, F Hatipoglu, B M Sumer \& J Fredsoe (2008): Wave boundary layer over a stone covered bed. Coastal Engineering, Vol 55, No 1, pp 1-20.

Fredsøe, J (1984): Turbulent boundary layers in wave current motion. J Hydr Eng, A S C E, Vol 110, Hy 10, pp 1103-1120.

Fredsøe, J \& R Deigaard (1992): Mechanics of coastal sediment transport. World Scientific, 369pp. Guard, P A (2010): Sheet flow sediment transport and swash hydrodynamics. PhD Thesis, Civil Engineering, The University of Queensland. Available from www.uq.edu.au/coastal.

Jensen, B L, B M Sumer \& J Fredsøe (1989): Turbulent oscillatory boundary layers at high Reynolds numbers. J Fluid Mech, Vol 206, pp 265-297.

Jonsson, I G \& N A Carlsen (1976): Experimental and theoretical investigations in an oscillatory turbulent boundary layer. J Hydraulic Res, Vol 14,pp 45-60.

Kamphuis, J W (1975): Friction factors under oscillatory waves. $J$ Waterways, Port, Coastal and Ocean Eng Div, A S C E, Vol 101, pp135-144.

Mathisen, P P \& O S Madsen (1999): Waves and currents over a fixed rippled bed. Bottom and apparent roughness for spectral waves and currents. J Geophys Res, Vol 104, No C8, pp 18447-18461.

Mirfenderesk, H \& I R Young (2003): Direct measurements of the bottom friction factor beneath surface gravity waves. Applied Ocean Research, Vol 25, pp 269-287.

Nielsen, P (1985): On the structure of oscillatory boundary layers. Coastal Eng, Vol 9, No 3, pp 261-276.

Nielsen, P (1992): Coastal Bottom Boundary Layers and Sediment Transport. World Scientific, 324pp.

Nielsen, P (2006): Sheet flow sediment transport under waves with acceleration skewness and boundary layer streaming. Coastal Engineering Vol 53, No 9, pp 749-758.

O’Donoghue, T, M Li, J Malarkey, S Pan, A G Davies, B A O’Connor (2004): Numerical and experimental study of wave generated sheet flow. Proc $29^{\text {th }}$ ICCE, Lisbon, World Scientific, pp 1690-1702. Riedel, H P (1972): Direct measurement of bed shear stress under waves. Ph D Thesis, Queens University, Kingston Ontario, 142pp.

Sleath, J F A (1987): Turbulent oscillatory flow over rough beds. J Fluid Mech, Vol 182, pp 369-409.

Van Doorn, Th (1982): Experimenteel onderzoek naar het snellheids-veld in de turbulente bodemgrenslaag in een oscillerende stroming in een golftunnel. TOW-Report M1562-1a. Delft Hydraulics Laboratories. 